\title{
Research on Drilling Scaling Unit Based on Virtual Prototyping
}

\author{
REN Hongwei \\ Marine engineering equipment research institute \\ Drilling Technology Research Institute \\ Dongying, China \\ Renhw_123@163.com \\ YIN Keping \\ College of mechanical and electronic engineering \\ China University of Petroleum (east China) \\ Tsingtao, China
}

\begin{abstract}
Current water pipeline drilling scaling equipment cannot clean effectively. In order to solve this problem, analyzes the dill descaling method and the characteristics of virtual prototyping technology, improved the unit. Construct three dimensional models with SolidWorks software, define subassembly relationship, on that basis complete virtual assembly of the overall unit, get virtual prototyping model, check assembly interference situation, provide the basis for subsequent design and experiment.
\end{abstract}

Keywords-Drilling scaling unit; Virtual prototyping; Threedimensional modeling; Virtual assembly

\section{INTRODUCTION}

Water pipeline is a kind of important oil extraction tools; usually the injection water has a poor quality, water pipeline scaling serious by prolonged use, result in a substantial diameter narrowed, water pressure drop, reduced water efficiency. Therefore water pipeline need regular cleaning. Current drilling scaling equipment cannot cleaning effectively, number of scaling pipe which cannot recycle, is facing scrap processing, which increased production costs. So the research on drilling scaling unit has important significance.

The traditional design method cycle is long, cost much on Product prototype and experimental, and can't form serialization, can't meet the requirements of modern machinery manufacturing industry. Virtual prototype technology can appear on the market fast, has a high quality and low cost, become the key to success in the fierce competition in the market of modern manufacturing enterprise.

\section{DRILl SCALING PRINCIPLE}

Processing object in conventional drilling process is metal, has many differences from scaling processing. Sum up for the following two points.

\author{
LIU Yancong \\ College of mechanical and electronic engineering \\ China University of Petroleum (east China) \\ Tsingtao, China
}

\author{
Li Sen \\ College of mechanical and electronic engineering \\ China University of Petroleum (east China) \\ Tsingtao, China
}

- Material of processing object is different. Metal belongs to the plastic material; the deposition is mixture of $\mathrm{CaCO} 3$, precipitation, silt, clay etc. Belongs to brittle material, has a different physical properties compared to metal. Drilling process bit force, drilling speed, chip formation way and clearance conditions are different. Compared with metal drilling, drilling scaling process is more similar to rock drilling process[3].

- Drilling object structure is different, water injection pipe itself has not be blocked off fully, left 10 40 mm unequal diameter hole, and hole wall is not smooth, drilling scaling should belong to the reamer.

When the bit do cutting motion in water pipeline scale surfaces with a certain amount of feed, bit blade applied a shear force to deposition, deposition induced a tensile stress with the sheer force, when the tensile stress more than the deposition tensile strength, deposition produce tiny crack, and when the crack increase, the deposition broke to pieces, take out by cutting fluid circulation, achieve the purpose of removing. This process is the process of drilling scaling. The process is leapfrog process; the deposition may produce two or three small rupture, and then broke to pieces. The whole process generally has four stages of deformation, occurrence crack, forming cutting nuclear, block split phase.

\section{THREE-Dimensional MODELING OF DRILLING SCALING UNIT}

Based on virtual prototype technology, establishment three dimensional model of drilling scaling unit by the use of software aided design function; implement virtual assembly and interference check. Provide foundation for the follow-up design and experiment. 


\section{A. Virtual Prototyping Technology}

Virtual design is a kind digital design method based on computer simulation model, has a broad prospect of application. The entire technical data in product development process can constitute virtual prototype, Engineering and technical personnel with it instead of physical prototype for air dynamics analysis, man-machine engineering research, collision test, etc. With the development of computer hardware and software technology and the progress of network technology, globalization, networking and virtualization has become an important feature of the development of the manufacturing industry. More and more enterprises in the new product development process using digital technology, and using the digital model complete design verification instead of many expensive entity[4]. Using virtual prototype replace physical prototype validates not only can shorten the development cycle, but also can improve the design efficiency[5]. In our country, the virtual prototype technology has been taking seriously in automotive, aerospace industry and other research fields[6].

Using virtual prototype can easily replace physical prototype, so as to avoid the high cost of actual model making and assembly, and the problem of difficulty in modification. Also shorten the development cycle, reduce cost.

\section{B. Components three dimension models}

\section{1) Bit system modeling}

SolidWorks has powerful functions, perfect data communication function, can virtual assembly automatically for users, correction scheme tips, and optional implementation scheme hint, etc. And can provide the virtualization of the development environment. In SolidWorks tool library, can undertake and real environment similar assembly and correction work, greatly improving the work efficiency[7].Using SolidWorks software modeling to drilling scaling unit, and real-time rendering, prepare for assembly.

Three dimensional models is composited by multiple characteristic element, characteristic refers to various single processing shape, which is generated by the sketch or menu commands, and characteristic element is the basic elements unit of parametric CAD software, also can be regarded as a kind of machining process.

On the basis the original drilling scaling unit, Modified the structure, get a new kind of drilling scaling unit. According to the new scaling bit, the feature of structure and the three dimensional modeling software needs, divided the bit into 4 parts of cutter body, guide, shock absorber, blade. The blade also can divide into external tooth, middle flat teeth, intermediate teeth and inner teeth four independent tooth. Intermediate teeth and inner teeth have the same structure.

Drill cutter body is the structure foundation; the rest parts of the structural unit are assembled on it. Drill cutter body modeling process can be seen as a machining process. In the modeling process, drill body can be seen as the sketch rotary get cylinder, the design parameters is the bit cutter body radius, inner hole radius, cone Angle, and the axial direction of the paragraphs size parameters. Using rotating order of SolidWorks software, get new drill cutter body $3 \mathrm{~d}$ model. This model is equivalent to the blank materials of machining, after a series of excision processing, can get qualified cutter body model, which is shown in figure1.

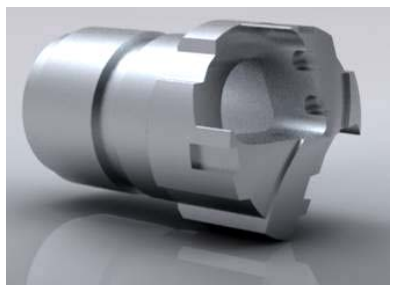

Figure 1. Drill cutter body model

According to the parent-child relationship of modeling characteristics, rotating feature called parent characteristics, the use of tensile excision command on the model in turn cut out of the inner clearance holes, blade tooth, guide groove, shock absorber tank and cutting fluid conveying tank, etc. As a child characteristics exist in the parent characteristics of the tree. Parent-child characteristics have joint relationship.

In the modeling process, in order to get the geometric constraint relation description of drill body, use of vertical, tangent, parallel geometric relation to define the drill body geometry shape.

Use the same method to establish blade $3 \mathrm{~d}$ model, which is shown in figure2.

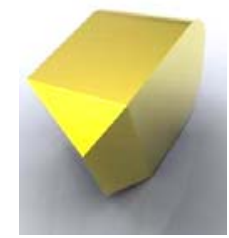

a. inner teeth

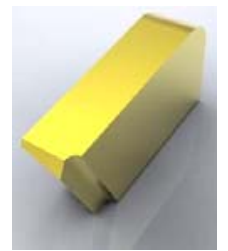

b. external teeth

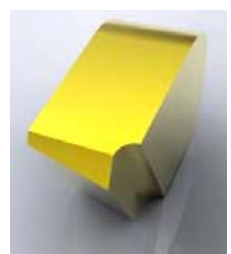

c. middle flat teeth
Figure 2. Drill cutters model

SolidWorks assembly function is specified by customer between the parts of the constraint relations, form assembly body. The forming assembly body can be used as a child assembly body, as one of the general assembly parts; can also take separate parts together constitute an assembly body[8].

In drill body assembly process, open the cutter body model as the parent parts and then insert the rest of the model, as a subcomponent. When insert a child components, this subcomponent file and assembly body file links, but subpart data also keep in a source document, so one pair component model for any change will update assembly body, guarantee model and assembly body unity.

The child components need assembly to bit cutter body model through the cooperation relationship. Use cooperate relationship can accurate positioning components, moving, rotating and operation, through the continue to add cooperate relationship, can adjust parts to the right position, Install blade, guide, shock absorber parts to bit knife body through the constraint relations. Cooperate relationship and bit body model as shown in figure 3, generated assembly body filename suffix for. SLDASM. 


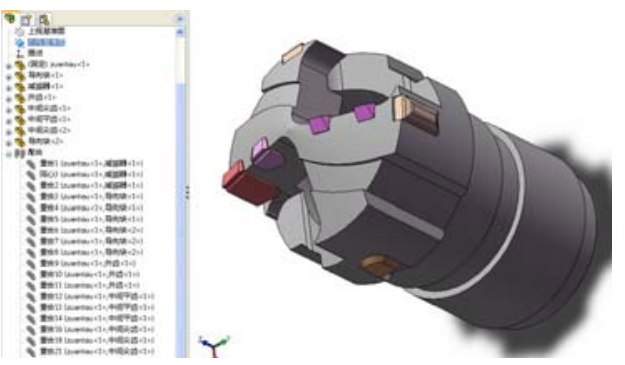

Figure 3. Drill system model

2) Drill pipe system three dimensional modeling

Drill pipe is thin rod, its characteristic in the end and connection negative pressure chip device crescent tank. When modeling can divided drill pipe into three parts, middle hollow cylinder, connection parts, and the crescent tank.

Use drawing tools to generate drill pipe middle cylinder, and with the geometric parameters for drill pipe are diameter, diameter, and length. Crescent tank using tensile excision method, because the crescent tank and pipe axis not vertical, but into $30^{\circ}$ Angle, so draw sketch before need to build the datum plane, operation excision command, the drill pipe model as shown in figure 4 .

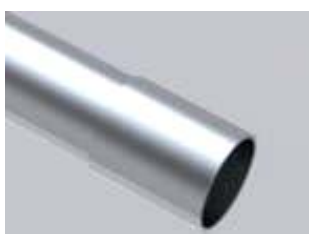

a. connection part

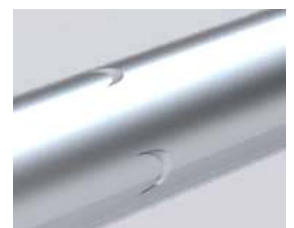

b. crescent tank
Figure 4. Drill pipe model

Drill pipe system including Drill stands, negative pressure chip removal device, etc., its model as shown in figure 5, figure 6.

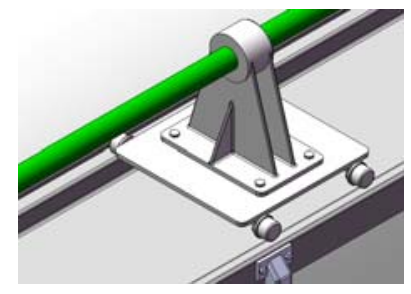

Figure 5. Drill stands

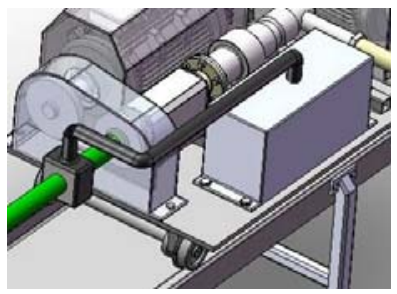

Figure 6. negative pressure chip removal device

\section{3) Overall unit virtual assembly}

On the basis of $3 \mathrm{~d}$ modeling of component, get overall unit virtual assembly, verify the correctness of the assembly design and operation, analysis design rationality effectively. Drilling scaling unit including bit system, power transmission system, drill pipe system and frame base system.

Bit system(see figure 7), which is the core block, function is to break the scale drilling, cutting fluid scour bit blade through conveying channel in the process, cooling lubrication, and carrying chip out.

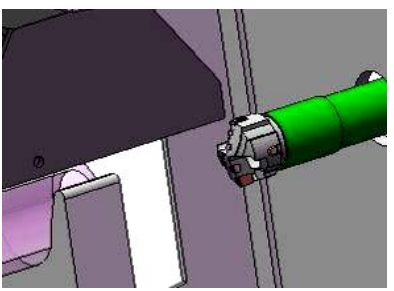

Figure 7. Bit system

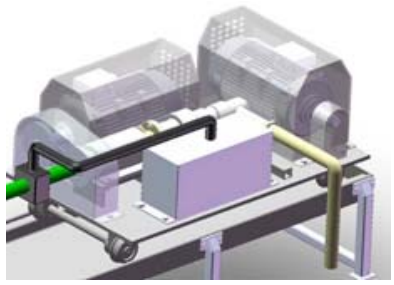

Figure 8. Power transmission system

Power transmission system(see figure8), Including motor, chain, reducer, etc. Motor main function is to drill rod rotation movement and feed movement with kinetic energy, chain drive will motor output kinetic energy transfer to reducer, reducer according to a certain proportion of motor speed for reduction, drive sprocket wheel rotation.

Drill pipe and chip removal system, the function is to take cutting fluid into pipeline with certain pressure, to produces negative pressure effect, accelerate the cutting fluid carrying chip successful, while preventing the chip into pipeline lead to a stuck drill problem.

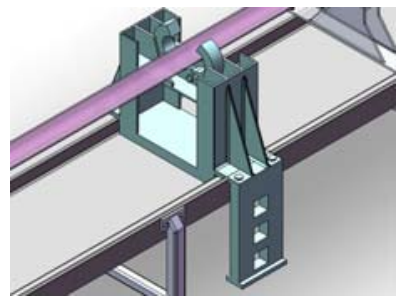

Figure 9. Pipe clamping device 


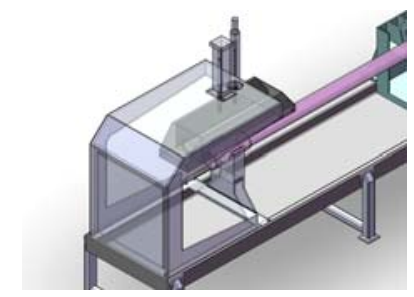

Figure 10. Preventer cover

Frame base system, including frame base, guide, pipe clamping device(see figure 9), preventer cover(see figure 10). Operations support supporting the whole system, and provide guide rail for drill pipe in feed movement, pipe clamping device clamping water pipeline, preventer cover prevent cutting fluid spill out.

Take frame base system as the parent components, read in other system as the child assembly body, use the insert - parts assembly body command, can read in each assembly body.

Define each child assembly body cooperation relationships, get virtual prototype model. Use interference check command complete interference, the calculation results shows in the dialog, interference parts will be displayed in red plot. After interference checking, did not find interference situation, cooperate relationship and whole model as shown in figure 11.

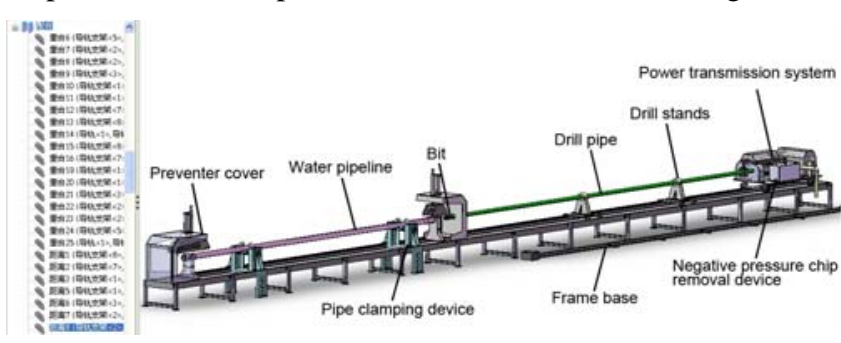

Figure 11. Drilling system virtual prototype

\section{CONCLUSION}

- Analysis of the drill scaling principle and processing object characteristics, improve the design on the basis of the original equipment, get new descaling bit.
- Introduces the concept and characteristics of virtual prototype technology, and applied to drill scaling system, improve the design efficiency.

- $\quad$ Build drill scaling unit three dimensional modeling by the use of CAD software, and get virtual assembly model, check assembly interference condition, provides the basis for the follow-up design and experiment.

\section{ACKNOWLEDGMENT}

I would like to express my gratitude to all those who helped me during the writing of this thesis. Thanks Professor Liu Yancong, who has offered me valuable suggestions, also thanks Yin Keping, with his help I can finish modeling. Last my thanks would go to my beloved family for their loving consideration and great confidence in me.

\section{REFERENCES}

[1] Chen Feng, Zhao Chunhui, and Chen Chaolin, "Oilfield water injection system scaling mechanism research,” Oil-Gas field Surface Engineering. Daqing, vol.25, pp7-8, July 2006.

[2] Xiao Yuantian, "Virtual manufacturing and the application of digital engineering in the car," Journal of System Simulation. Beijing,pp342347, March 2002.

[3] Zhu Xiaohua, Deng Fucheng,Jia Yanjie,TongHua,Xu Jianmin, "PDC bit flow field numerical simulation and hydraulic structure optimization," Petroleum machinery. Wuhan,Vol. 38, pp1-4, August 2010.

[4] Yu Weiling,Wan Jun, "Virtual manufacturing technology (VMT) development and applied research,” Manufacturing Automation. Beijing, pp1-4, February 2008.

[5] Sun Fuzhen, "Mechanical virtual reality technology application and development,” Machinery Design \& Manufacture. Shenyang, pp264266, May 2010.

[6] Cheng Yunzhang,Feng Jinzhi,Lu Xi, "Virtual prototype technology pump mechanical vibration research,” Machinery Design \& Manufacture. Shenyang, pp114-116, April 2009.

[7] Chen Hua, "Based on Solidworks car doors and Windows elevator riveting pressure platform design,” Machinery Design \& Manufacture. Shenyang, pp254-255, August 2010.

[8] Zorriassatine F, Wykes C, Parkin R, et al. "A survey of virtual prototypingtechniques for mechanical product development. Proceedings of IMech,” E.PartB : J. Engineering Manufacture, pp 513-530, Jan. 2003. 